\title{
Impact of a quality improvement programme on family planning services in Egypt
}

R. Hong, ${ }^{7}$ V. Mishra ${ }^{2}$ and N. Fronczak ${ }^{3}$

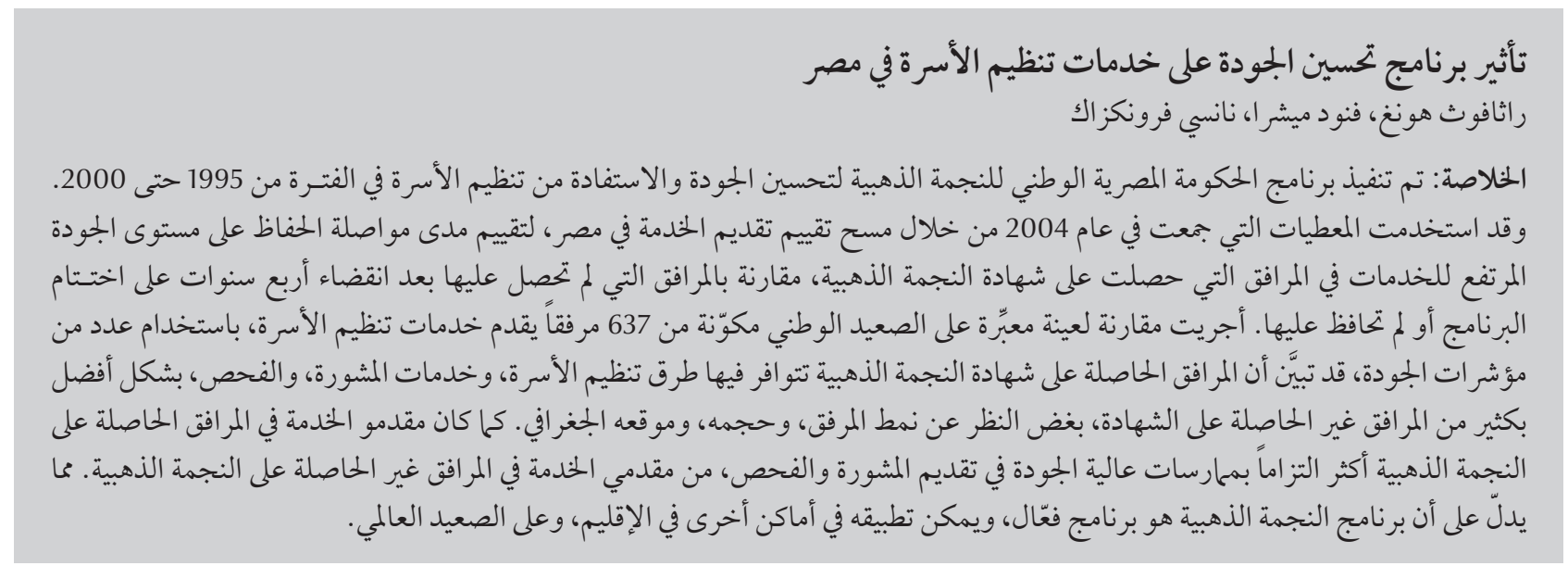

ABSTRACT The Egyptian government's national Gold Star programme to improve the quality and usage of family planning was conducted from 1995 to 2000. Data from the 2004 Egypt Service Provision Assessment survey were used to assess whether the certified Gold Star facilities had sustained higher quality services than non-Gold Star facilities 4 years after conclusion of the programme. A nationally representative sample of 637 facilities providing family planning services were compared using several quality indicators. Gold Star facilities had significantly better availability of family planning methods, counselling and examination services than non-Gold Star facilities, independent of type, size and geographical location. Providers in Gold Star facilities were also more likely to adhere to higher quality practices in counselling and examination than in non-Gold Star facilities. The Gold Star programme was effective and could be implemented elsewhere in the region and globally.

\section{Impact d'un programme d'amélioration de la qualité sur les services de planification familiale en Égypte}

RÉSUMÉ Le programme national d'excellence Gold Star du gouvernement égyptien pour l'amélioration de la qualité des services de planification familiale et de leur utilisation a été mené de 1995 à 2000. Les données de l'enquête sur l'évaluation des prestations de service en Égypte datant de 2004 ont été utilisées pour savoir si les établissements certifiés Gold Star avaient fourni des services de qualité supérieure par rapport à ceux qui ne l'étaient pas, quatre ans après l'achèvement du programme. Dans un échantillon national représentatif, 637 établissements fournissant des services de planification familiale ont été comparés à l'aide de plusieurs indicateurs de qualité. Les établissements certifiés Gold Star offraient un niveau de disponibilité supérieur pour les méthodes de planification familiale, les services de conseil et les examens par rapport aux autres établissements, indépendamment du type d'établissement, de sa taille ou de sa situation géographique. Les prestataires dans les établissements certifiés Gold Star étaient plus susceptibles d'observer des pratiques de qualité supérieure en termes de conseils et d'examens que les autres. Le programme de certification Gold Star s'est révélé efficace et pourrait être mis en œuvre ailleurs dans la Région et dans le monde.

'Measure Demographic and Health Survey, ICF Macro, Calverton, Maryland, United States of America (Correspondence to R. Hong: rhong@icfi. com).

${ }^{2}$ Population Policy Section, Population Division, DESA, United Nations, New York, New York, United States of America.

${ }^{3}$ Social Sectors Development Strategies, Boston, Massachusetts, United States of America.

Received: 31/05/09; accepted: 04/08/09 


\section{Introduction}

Egypt's rapid population growth in recent decades is considered a major obstacle to the developmental goals set by the Egyptian government [1]. For more than 2 decades, the Egyptian government in collaboration with various international donor agencies has been pursuing a national population control programme with the objectives to increase contraceptive use and to reduce fertility. As a consequence, between 1980 and 2003, current use of modern contraceptive methods among married women increased from $23 \%$ to $57 \%$ and the total fertility rate decreased from 5.3 to 3.2 children per woman [2]. Despite the progress, in 2002, according to the Human Development Report, Egypt had a population of 71 million growing at an annual rate of $1.9 \%$ [3].

Following the United Nations International Conference on Population and Development in 1994 in Cairo, which identified providing high quality family planning services as an essential component of any reproductive health programme [4], Egypt's Ministry of Health and Population and Ministry of Information with support from the United States Agency for International Development developed a national family planning quality improvement programme, called the Gold Star programme [5]. The programme was designed to promote the supply of quality family planning services through better training and supervision of providers and to stimulate demand for family planning by promoting higher quality services to the public [6]. The programme assessed each facility in Egypt providing family planning services on a comprehensive checklist of 101 indicators of quality, including indicators of infrastructure availability, supply of family planning methods and condition of the facility. The assessment was undertaken quarterly to ensure that quality was sustained. A facility that met all 101 quality criteria for 2 consecutive quarters was awarded a Gold Star certificate, which had to be maintained at successive quarterly evaluations to retain the status. The Gold Star programme was concluded in $2000[7]$.

Previous research has linked the availability of a broad range of contraceptive methods to increased utilization $[8,9]$. Better family planning infrastructure and adherence of providers to standard practices - discussing issues and side-effects of contraceptive methods, protecting client privacy, evaluating reproductive and medical history and conducting basic examinations to ensure safe administration of the methods-have also been linked with greater acceptance of family planning [10-13].

Using data from a recent national health facility survey in Egypt, this study assessed whether the certified Gold Star facilities had sustained higher quality services than non-Gold Star facilities 4 years after the conclusion of the programme. The study compared Gold Star and non-Gold Star facilities on several indicators of quality of family planning services: availability of necessary infrastructure, availability of a broad range of family planning methods, adequately trained staff, adherence to standard practices in counselling and examination and systems to ensure safe and effective administration of the family planning methods.

\section{Methods}

The data reported in this paper are part of an Egypt Service Provision Assessment (EPSA) survey conducted in 2004. The ESPA survey collected information from a nationally representative sample of health facilities and health service providers. The primary objective of that survey was to provide national and regional estimates of facility characteristics and their capacity to provide quality services for family planning, maternal and child health, sexually transmitted infections, HIV/AIDS and other infectious diseases, such as malaria and tuberculosis.

\section{Sample}

Data were collected from a sample of 659 health facilities. Private, for-profit health facilities were not included in the sample. The analysis in this paper was limited to 637 of the sample facilities that provided family planning services and 1930 observations of consultations between family planning providers and clients. The survey also identified which facilities had been certified as Gold Star during 1995-2000. Further details about the survey design are provided in the main ESPA survey report [14].

\section{Data collection}

\section{Family planning quality index}

Using ESPA data from facility inventories and provider interviews, 4 major dimensions of quality of family planning care were identified:

- Contraceptive supply: provision for and availability of each of the 5 main family planning methods [oral contraceptives, injectables, condoms, subdermal implants (Norplant ${ }^{\circ}$ ) and intrauterine contraceptive devices (IUDs)], as well as the provision of education and information on the rhythm method.

- Counselling: availability of standard guidelines, privacy in counselling room, visual aids and an individual client family planning card.

- Examination: availability of a private room for examinations, soap, running water, clean latex gloves, disinfecting solution, sharps box, examination table or bed, examination light and vaginal speculum.

- Management: whether at least 50\% of the staff received training in family planning in the last 12 months; whether at least $50 \%$ of the staff received supervision in the last 6 months; and whether the facility had 
an up-to-date family planning register for the last 7 days.

The family planning quality index was used to compare the Gold Star and non-Gold Star facilities on each of the 4 dimensions of quality and on overall quality after statistically controlling for facility type [hospital, maternal and child health $(\mathrm{MCH})$ /urban health unit, rural health unit, health office/mobile unit, nongovernmental organization (NGO)]; size of facility (small: $<10$ staff, medium: 10-19 staff, large: 20+ staff); and geographical location of facility (urban governorate, lower Egypt, upper Egypt). A list of the governorates included in each region is provided in the footnote to Table 1 .

\section{Adherence to standard practices}

The study also usedEPSA data on observations of provider-client interactions to assess adherence to standard practices in counselling and examination. The indicators for this domain were: whether the provider ensured visual privacy; ensured auditory privacy; assured confidentiality; reviewed the client card; recorded information on the client card; used visual aids during the consultation; and discussed the next visit. Again, Gold Star and non-Gold Star facilities were compared on the 7 indicators,

\section{Ethical issues}

The findings presented here are based on an analysis of existing survey data with all identifier information removed. Informed consent was obtained from all respondents in the survey before asking questions.

\section{Analysis}

For each facility, scores on the items from the 4 dimensions of quality of family planning care were added and the total score was standardized to 25 . An overall index of quality of family planning services was calculated by adding the individual index scores for the 4 dimensions (total score range $0-100$ ).

The bivariate comparisons between Gold Star and non-Gold Star facilities on various items of quality offamily planning services were made using the chisquared statistic with significance levels. Multivariate analysis was conducted using multiple regression using STATA statistical package, version 8.0. Certain types of facilities were over-sampled and in all analyses sample weights were used to restore the representativeness of the facilities.

\section{Results}

\section{Distribution of Gold Star and non-Gold Star facilities}

Table 1 shows the distribution of family planning facilities by type, size and geographical location. One-half of the facilities were rural health units and only one-tenth were hospitals. Two-fifths of the facilities had $<10$ staff members, and about one-third had 20+ staff. About one-half of the facilities were in lower Egypt.

Table 1 also shows the proportion of the facilities that were certified as Gold
Star facilities at some time during 1995 and 2000. Overall, $16.7 \%$ of all family planning facilities had received Gold Star certification. Rural health units were most likely (47.7\%) and NGOs were least likely (3.6\%) to have received the certification. Larger facilities and facilities in lower Egypt were more likely to have received the Gold Star certification than other facilities.

\section{Comparison of family planning quality indicators}

Table 2 shows that Gold Star facilities provided higher quality family planning services for the majority of items in each of the 4 family planning quality dimensions than did non-Gold Star facilities.

The Gold Star facilities were significantly more likely to provide each of the contraceptive methods than nonGold Star facilities. The difference was particularly evident for discussion of the rhythm method, but the difference was not significant in the case of IUD provision.

\begin{tabular}{|c|c|c|c|c|}
\hline \multirow[t]{2}{*}{ Characteristic } & \multicolumn{2}{|c|}{ Total } & \multirow{2}{*}{$\begin{array}{c}\text { Gold star } \\
\%\end{array}$} & \multirow{2}{*}{$\begin{array}{c}\text { Non-Gold star } \\
\%\end{array}$} \\
\hline & No. & $\%$ & & \\
\hline \multicolumn{5}{|l|}{ Facility type } \\
\hline Hospital & 67 & 10.4 & 10.5 & 89.5 \\
\hline $\mathrm{MCH} /$ urban health unit & 96 & 15.0 & 32.4 & 67.6 \\
\hline Rural health unit & 319 & 50.1 & 47.7 & 52.3 \\
\hline Health office/mobile unit & 83 & 13.1 & 5.9 & 94.1 \\
\hline NGO & 73 & 11.4 & 3.6 & 96.4 \\
\hline \multicolumn{5}{|l|}{ Size of facility ${ }^{a}$} \\
\hline Small & 258 & 40.8 & 8.4 & 91.6 \\
\hline Medium & 173 & 27.4 & 19.4 & 80.6 \\
\hline Large & 201 & 31.8 & 26.2 & 73.8 \\
\hline \multicolumn{5}{|l|}{ Region $^{\mathrm{b}}$} \\
\hline Urban governorate & 71 & 11.2 & 11.0 & 89.0 \\
\hline Lower Egypt & 312 & 49.0 & 23.5 & 76.5 \\
\hline Upper Egypt & 254 & 39.8 & 11.5 & 88.5 \\
\hline Total & 637 & 100.0 & 16.7 & 83.3 \\
\hline
\end{tabular}

${ }^{a}$ Small < 10 staff; medium: $10-19$ staff; large: $20+$ staff.

${ }^{b}$ Urban governorates: Cairo, Alexandria, Port Said and Suez; lower Egypt: Damietta, Dakahlia, Sharkia, Kayubia, Kafr-el-Sheikh, Gharbia, Menoufia, Behera and Ismailia; upper Egypt: Giza, Beni Suef, Fayoum, Menya, Assiut, Sohag, Qena, Aswan and Luxor.

$\mathrm{MCH}=$ maternal and child health; $\mathrm{NGO}=$ nongovernmental organization. 


\begin{tabular}{|c|c|c|c|c|}
\hline Dimension/Indicator & $\begin{array}{c}\text { Total } \\
\% \\
(n=637)\end{array}$ & $\begin{array}{c}\text { Gold star } \\
\% \\
(n=110)\end{array}$ & $\begin{array}{c}\text { Non-Gold star } \\
\% \\
(n=527)\end{array}$ & $P$-value \\
\hline \multicolumn{5}{|l|}{ Contraceptive supply } \\
\hline Oral contraceptives & 92.7 & 99.4 & 91.3 & 0.004 \\
\hline Injectables & 96.1 & 100.0 & 95.3 & 0.020 \\
\hline Condoms & 86.6 & 94.7 & 84.9 & 0.006 \\
\hline Norplant ${ }^{\circledR}$ & 12.5 & 18.3 & 11.3 & 0.043 \\
\hline IUDs & 98.0 & 100.0 & 97.6 & 0.099 \\
\hline Rhythm & 66.8 & 82.1 & 63.6 & $<0.001$ \\
\hline \multicolumn{5}{|l|}{ Counselling } \\
\hline Guidelines & 36.6 & 54.8 & 32.8 & $<0.001$ \\
\hline Privacy in counselling room & 86.0 & 86.4 & 85.9 & 0.895 \\
\hline Visual aids & 89.3 & 94.4 & 88.2 & 0.058 \\
\hline Individual client card & 87.1 & 95.6 & 85.3 & 0.003 \\
\hline \multicolumn{5}{|l|}{ Examination } \\
\hline Private room & 84.1 & 78.5 & 85.3 & 0.072 \\
\hline Soap & 67.2 & 82.3 & 64.1 & $<0.001$ \\
\hline Running water & 85.6 & 86.7 & 85.4 & 0.709 \\
\hline Clean latex gloves & 30.0 & 39.5 & 28.1 & 0.017 \\
\hline Disinfecting solution & 87.9 & 95.0 & 86.4 & 0.012 \\
\hline Sharps box & 68.7 & 88.1 & 64.6 & $<0.001$ \\
\hline Examination table/bed & 98.1 & 100.0 & 97.7 & 0.105 \\
\hline Examination light & 91.7 & 94.9 & 91.0 & 0.182 \\
\hline Vaginal speculum & 92.2 & 97.5 & 91.1 & 0.022 \\
\hline \multicolumn{5}{|l|}{ Management } \\
\hline $\begin{array}{l}>50 \% \text { of staff received training in } \\
\text { family planning in last } 12 \text { months }\end{array}$ & 28.6 & 27.1 & 29.0 & 0.740 \\
\hline $\begin{array}{l}>50 \% \text { of staff received supervision in } \\
\text { last } 6 \text { months }\end{array}$ & 93.1 & 97.8 & 92.2 & 0.023 \\
\hline $\begin{array}{l}\text { Complete family planning registration } \\
\text { for last } 7 \text { days }\end{array}$ & 90.6 & 100.0 & 88.6 & $<0.001$ \\
\hline
\end{tabular}

$n=$ number offacilities; IUD = intrauterine contraceptive device.

For counselling indicators, availability of guidelines was significantly better in Gold Star than non-Gold Star facilities, but there was no significant difference between the 2 types of facilities in providing privacy in the counselling room.

Gold Star facilities were also significantly more likely to have most of the examination room supplies than non-Gold Star facilities, except there was no significant difference in the availability of private room, running water, examination table/bed and examination light.
A significantly higher proportion of Gold Star facilities than non-Gold Star facilities had complete family planning registers for the last 7 days and had supervised at least half of their staff in the last 12 months. There was no significant difference between the 2 types of facilities on the family planning training indicator; although slightly fewer Gold Star facilities staff had trained more than half their staff in the last 12 months this was not statistically significant.

Table 3 shows the average family planning quality index scores for each quality dimension-contraceptive supply, counselling, examination and management - for Gold Star and nonGold Star facilities. The Gold Star facilities had a higher mean score on each individual dimension of quality of family planning services. Overall, the Gold Star facilities had a mean family planning quality score of 80.4 compared with 72.7 for non-Gold Star facilities.

Results from the bivariate analysis indicated that the Gold Star facilities had significantly higher family planning quality index scores than non-Gold Star facilities $(P<0.01)($ Table 4$)$. Even 


$\begin{aligned} & \text { Table } 3 \text { Comparison of Gold Star and non-Gold Star facilities on the } 4 \text { dimensions } \\
& \text { of the family planning quality of care index }\end{aligned}$
\begin{tabular}{lccc}
\hline Dimension & Maximum score & $\begin{array}{c}\text { Gold Star } \\
(\boldsymbol{n}=\mathbf{1 1 0})\end{array}$ & $\begin{array}{c}\text { Non-Gold Star } \\
(\boldsymbol{n}=527)\end{array}$ \\
& & Mean (SE) & Mean (SE) \\
Contraceptive supply & 25 & $19.9(0.30)$ & $18.0(0.18)$ \\
Counselling & 25 & $20.5(0.44)$ & $18.0(0.24)$ \\
Examination & 25 & $21.2(0.36)$ & $19.3(0.18)$ \\
Management & 25 & $18.7(0.44)$ & $17.5(0.25)$ \\
Total & 100 & $80.4(0.84)$ & $72.7(0.59)$ \\
\hline
\end{tabular}

$n=$ number offacilities; $S E=$ standard error .

when controlling for type, size and geographic location of facility in the multivariate analysis, the difference in the overall family planning quality index between the Gold Star and non-Gold Star facilities remained large and statistically significant $(P<0.01)$ (Table
4). When analysed separately for individual dimensions of quality, the Gold Star facilities had significantly higher adjusted index scores than non-Gold Star facilities for contraceptive supply, counselling and examination, but not for management.

\section{Comparison of provider adherence to standard practices indicators}

Table 5 compares the Gold Star and non-Gold Star facilities on 7 indicators of provider adherence to standard practices in counselling and examination based on observed provider-client interactions. Providers in Gold Star facilities were significantly more likely to adhere to standard practices in counselling and examination than those in non-Gold Star facilities on 6 of the 7 indicators.

\section{Discussion}

The ESPA surveys collect valuable national and regional information on

\begin{tabular}{|c|c|c|c|c|c|}
\hline Characteristic & Contraceptive supply & Counselling & Examination & Management & Total \\
\hline \multicolumn{6}{|l|}{ Bivariate analysis } \\
\hline \multicolumn{6}{|l|}{ Gold Star status } \\
\hline Non-Gold Star ${ }^{\mathrm{a}}$ & - & - & - & - & - \\
\hline Gold Star & $1.97^{* *}$ & $2.52^{* *}$ & $1.91^{* *}$ & 1.27 & $7.63^{* *}$ \\
\hline \multicolumn{6}{|l|}{ Multivariate analysis } \\
\hline \multicolumn{6}{|l|}{ Gold Star status } \\
\hline Non-Gold Star ${ }^{\mathrm{a}}$ & - & - & - & - & - \\
\hline Gold Star & $1.16^{*}$ & $1.46^{*}$ & $1.3^{*}$ & 0.65 & $4.57^{* *}$ \\
\hline \multicolumn{6}{|l|}{ Facility type } \\
\hline Hospital $^{\mathrm{a}}$ & - & - & - & - & - \\
\hline $\mathrm{MCH} /$ urban health unit & 0.06 & -1.38 & 0.07 & 0.02 & -1.24 \\
\hline Rural health unit & -0.84 & $-1.47^{*}$ & -0.93 & -0.52 & $-3.76^{*}$ \\
\hline Health office/mobile unit & -1.13 & $-4.17^{* *}$ & $-2.17^{*}$ & -0.06 & $-7.53^{* *}$ \\
\hline NGO & $-4.49^{* *}$ & $-6.55^{* *}$ & $-1.77^{*}$ & $-8.12^{* *}$ & $-20.93^{* *}$ \\
\hline \multicolumn{6}{|l|}{ Size offacility } \\
\hline Small ${ }^{a}$ & - & - & - & - & - \\
\hline Medium & -0.59 & -0.27 & -0.01 & -0.76 & -1.63 \\
\hline Large & -0.51 & 0.39 & 0.61 & -0.43 & 0.07 \\
\hline \multicolumn{6}{|l|}{ Geographic region } \\
\hline Urban governorate ${ }^{a}$ & - & - & - & - & - \\
\hline Lower Egypt & $1.76^{* *}$ & 0.19 & -0.57 & 0.16 & 1.54 \\
\hline Upper Egypt & -0.06 & -0.82 & -0.83 & 0.32 & -1.39 \\
\hline Constant & $18.67^{* *}$ & $20.55^{* *}$ & $20.72^{* *}$ & $18.94^{* *}$ & $78.89^{* *}$ \\
\hline
\end{tabular}

${ }^{a}$ Reference group

${ }^{*} P<0.05$; ${ }^{*} P<0.01$.

$n=$ number of facilities; $M C H=$ maternal and child health; $N G O=$ nongovernmental organization . 


\begin{tabular}{|c|c|c|c|c|}
\hline Indicator & $\begin{array}{c}\text { Total \% } \\
(n=1930)\end{array}$ & $\begin{array}{l}\text { Gold Star \% } \\
\qquad(n=436)\end{array}$ & $\begin{array}{l}\text { Non-Gold Star \% } \\
\qquad(n=1493)\end{array}$ & $P$-value \\
\hline Ensured visual privacy & 74.5 & 85.2 & 71.4 & $<0.001$ \\
\hline Ensured auditory privacy & 73.9 & 84.9 & 70.7 & $<0.001$ \\
\hline Assured about confidentiality & 20.0 & 32.3 & 16.4 & $<0.001$ \\
\hline Reviewed individual client card & 65.9 & 72.9 & 63.9 & $<0.001$ \\
\hline Wrote on individual client card & 75.9 & 83.2 & 73.7 & $<0.001$ \\
\hline Used visual aids during consultation & 7.3 & 10.9 & 6.3 & $<0.001$ \\
\hline Discussed next visit & 78.3 & 76.9 & 78.8 & 0.399 \\
\hline
\end{tabular}

$n=$ number of provider-client interactions.

health care facility characteristics and their capacity to provide family planning, maternal and child health, HIV/ AIDS and other services. The surveys provide important baseline information about the health care delivery systems, which can be used to evaluate various health and family welfare programme interventions.

Using information on family planning facilities collected in the 2004 ESPA survey, the study reported here evaluated whether the facilities that were certified as Gold Star during 1995-2000 sustained higher quality services than the facilities that were never certified as such. The results show that the Gold Star facilities continued to provide higher quality family planning services on most quality indicators than non-Gold Star facilities 4 years after the conclusion of the programme. The Gold Star facilities provided significantly higher quality services independently of the type, size and geographic location of facilities. The results also show that providers in the Gold Star facilities were significantly more likely to adhere to standard practices in counselling and examination of family planning clients than those in non-Gold Star facilities.

There are several limitations of this analysis that should be kept in mind when interpreting the results. First, there was no initial comparison of the Gold
Star and non-Gold Star facilities at the conclusion of the Gold Star programme in 2000. Therefore, it is not possible to evaluate how the differences in the quality of services between the 2 types of facilities have changed over time.

Another weakness is that the ESPA sample excluded private, for-profit family planning facilities. Although private, for-profit facilities were also excluded from the government's Gold Star programme, it would have been useful to compare the Gold Star facilities with non-Gold Star facilities in both the public and private sectors.

Thirdly, our comparison of quality indicators between the Gold Star and non-Gold Star facilities was limited to the information collected in the survey, which was not identical to the 101 indicators of quality that were originally used to identify Gold Star facilities. Moreover, there are several other important aspects of quality, such as waiting time and client respect, that are not included in our analysis.

Finally, while the facilities in the ESPA sample were randomly selected, the observations of provider-client interactions were based on a convenient, opportunistic sample of clients who happened to visit the facility on the day of data collection. This may have introduced some bias, but to the extent that data collection days and the times of visit were random across the facilities, any effect of this bias on the overall comparison of Gold Star and non-Gold Star facilities is likely to be small.

Notwithstanding these limitations, there is enough evidence to show that the Gold Star programme was among the successful family planning and population programmes in Egypt. The programme improved quality of care in family planning in Egypt by introducing elements of quality concepts and practices including supplying good quality services, creating demand for quality and research and evaluation of quality. The Gold Star programme was not only effective but also simple in concept and could therefore be implemented elsewhere in the region and globally.

\section{Acknowledgements}

The authors acknowledge Gulnara Semenov for providing valuable information about the Gold Star programme. Funding for this research was provided by the United States Agency for International Development through the MEASURE DHS project (\# GPO-C00-03-00002-00). The views presented in the paper do not represent the views of the United States Agency for International Development or the organizations to which the authors are affiliated. 


\section{References}

1. Ali KA. Modernization and family planning programs in Egypt. Middle East Report (New York, NY), 1997, 27:40-44.

2. Human development report 2004: Cultural liberty in today's diverse world. New York, United Nation Development Program, 2004.

3. Egypt interim demographic and health survey 2003. Calverton, Maryland, ORC Macro, 2004.

4. Summary of the ICPD programme of action. United Nations Population Fund [wesbsite] (http://www.un.org/ecosocdev/ geninfo/populatin/icpd.htm, accessed 1 November 2010).

5. Egypt activities datasheet 2001-02. United States Agency for International Development [wesbsite] (http://www.usaid.gov/ pubs/cbj2002/ane/eg/263-020.html accessed 1 November 2010).

6. John Hopkins University/Center for Communication Program. Egypt's Gold Star quality program wins clients and communities. Communication Impact, November 1998, Number 4 (http://www.jhuccp.org/pubs/ci/4/4.pdf, accessed 18 October 2010).

7. Best practices in accreditation. Best practices update: Reproductive health, family planning and HIV/AIDS. Arlington, Virginia, Advance Africa, 2004.
8. Greenspan A. Adding choice to the contraceptive mix: lessons from Indonesia. Asia-Pacific Population and Policy, 1991, 19:1-4.

9. Greenwell KF. Contraceptive method mix menu: providing healthy choices for women. World Health Statistics Quarterly, 1996, 49:88-93.

10. Brown L et al. Quality of care in family planning services in Morocco. Studies in Family Planning, 1995, 26:154-168.

11. Gilson L, Magomi M, Mkangaa E. The structural quality of Tanzanian primary health facilities. Bulletin of the World Health Organization, 1995, 73:105-114.

12. Bertrand J. The EVALUATION Project promotes consensus on family planning quality indicators. QA Brief, 1994, 3:6-7.

13. Askew I, Mensch B, Adewuyi A. Indicators for measuring the quality of family planning services in Nigeria. Studies in Family Planning, 1994, 25:268-283.

14. Egypt service provision assessment survey 2004. Calverton, Maryland, ORC Macro, 2005.

\section{Medical eligibility criteria for contraceptive use, 4th edition}

Medical eligibility criteria for contraceptive use reviews the medical eligibility criteria for use of contraception, offering guidance on the safety of use of different methods for women and men with specific characteristics or known medical conditions. The recommendations are based on systematic reviews of available clinical and epidemiological research. It is a companion guideline to Selected practice recommendations for contraceptive use. Together, these documents are intended to be used by policy-makers, programme managers, and the scientific community, to support national programmes in the preparation of service delivery guidelines.

The fourth edition has been fully updated and expanded. It includes over 86 new recommendations and 165 updates to recommendations in the previous edition. To assist users familiar with the third edition, new and updated recommendations are highlighted. Everyone involved in providing family planning services and contraception should have the fourth edition of Medical Eligibility Criteria for Contraceptive Use to hand.

This publication is currently in press but is expected to be available later this month (January 2011). Further information about this and other WHO publication is available at: http://www.who.int/publications/en/ 